\title{
Diferencias en el servicio y resto en el top- 8 del ranking masculino y femenino de
} tenis

\author{
Bernardino Javier Sánchez-Alcaraz Martínez, Javier Courel-Ibáñez, Alejandro Sánchez- \\ Pay y Samuel García Cambronero
}

\author{
Universidad de Murcia, España
}

\section{RESUMEN}

El objetivo de este trabajo será analizar los parámetros de rendimiento al servicio y resto en el ranking del top-8 masculino y femenino de tenis durante el año 2017.Se recogieron las estadísticas de rendimiento al saque y al resto en torneos ATP y WTA de los 8 primeros jugadores y jugadoras del ranking durante el año 2017.Los resultados mostraron que el top-8 masculino y femenino gana un porcentaje mayor de puntos y juegos al saque que al resto, además de ganar más puntos al primer servicio que al segundo. El ranking masculino registró valores más elevados los parámetros de saque, mientras que el ranking femenino registró un mejor rendimiento en el resto. Finalmente, los jugadores masculinos con una mejor posición en el ranking tienen mayor porcentaje de break points salvados y puntos ganados al saque, aspecto que no parece suceder en el ranking femenino. Los resultados de este estudio ayudan a conocer las diferencias en los parámetros de saque y resto entre el tenis masculino y femenino.
Palabras clave: Análisis del rendimiento, Tenis profesional, Saque, Resto, Ranking.

Recibido: 30 Mayo 2018

Aceptado:5 Julio 2018

Autor correspondiente:

Bernardino Javier Sánchez-

Alcaraz Martínez, Universidad

de Murcia, España.

Correo electrónico:

Bjavier.sanchez@um.es

\section{INTRODUCCIÓN}

El análisis de la competición tiene como objetivo registrar y analizar comportamientos y acciones de los deportistas en situaciones reales de juego (O'Donoghue, Girad, \& Reid, 2013). El tenis es el deporte de raqueta donde más se ha aplicado el análisis del rendimiento o competición (O’Donoghue \& Ingram, 2001), definiéndose una serie de variables o indicadores de rendimiento que contribuyen el éxito en la competición (Hughes \& Franks, 2004). Entre todos estos indicadores, el saque o servicio es a menudo considerado como el aspecto más crítico de un jugador, y diferentes estudios han afirmado que es el golpe más determinante en el resultado de un partido de tenis (Giampolo \& Levey, 2013).En este sentido, Barnett, Meyer \& Pollard (2008), encontraron que los 100 mejores jugadores del ranking masculino ganaron casi el $80 \%$ de los juegos al servicio y el $22 \%$ de los juegos al resto, sin existir diferencias por ranking. Por otro lado, os puntos ganados con el segundo servicio y los puntos ganados al resto en el segundo servicio son predictores significativos de la parte superior del ranking en el top 100 de clasificación profesional. Sin embargo, no existen trabajos que comparen las diferencias en estas variables entre el tenis masculino y femenino, ni su influencia en la clasificación mundial. Por lo tanto, el objetivo de este trabajo será analizar los parámetros de rendimiento al servicio y resto en el ranking del top-8 masculino y femenino de tenis durante el año 2017

\section{MÉTODO \\ Muestra}

La muestra de la investigación estuvo compuesta por un total de 16 jugadores, pertenecientes al top- 8 del ranking de tenistas masculinos (Edad: 27,3 $\pm 4,2$ años; Altura: 189,6 \pm 7,8 $\mathrm{cm}$ ) y femeninas (Edad: $25,9 \pm 4,2$ años; Altura: $174,4 \pm 6,5 \mathrm{~cm}$ ) del circuito profesional de tenis.

\section{Procedimiento}

Se recogieron las estadísticas de competición en torneos ATP y WTA de los 8 primeros jugadores y jugadoras del ranking al finalizar el año 2017. Los datos fueron seleccionados de la información publicada en la Web Oficial ATP (www.atpworldtour.com/en/stats) y (www.wtatennis.com/stats). Las variables seleccionadas para el 
rendimiento en el servicio fueron: \% primer servicio, \% puntos ganados con el primer saque, \% puntos ganados con el segundo saque, \% de puntos de break salvados al saque, \% juegos ganados al saque y $\%$ de puntos ganados al saque. Las variables seleccionadas para el rendimiento en resto fueron: \% puntos ganados al resto en el primer saque, $\%$ puntos ganados al resto en el segundo saque, \% de puntos de break ganados, $\%$ juegos ganados al resto y $\%$ de puntos ganados al resto.

\section{Análisis de datos}

Se realizó una comparación de medias entre sexos (masculino vs femenino) utilizando la prueba T-Student. Posteriormente, se realizó un análisis de regresión lineal por pasos para identificar los parámetros con mayor influencia sobre la posición en el ranking, tanto en masculino como en femenino. La significación se estableció en $p<.05$. Todos los datos fueron analizados con el paquete estadístico IBM SPPS 20.0 para Windows (Armonk, NY: IBM Corp.).

\section{RESULTADOS}

La tabla 1 muestra los resultados de las comparaciones de las medias de los parámetros de rendimiento en saque y resto entre el top-8 masculino y femenino. El ranking masculino registró valores más elevados en los parámetros de saque, mientras que el ranking femenino registró un mejor rendimiento en el resto. El porcentaje de acierto del primer saque fue similar para ambos sexos.

\begin{tabular}{|c|c|c|c|c|}
\hline Variable & Masculino & Femenino & Diff. & p \\
\hline \multicolumn{5}{|l|}{ Rendimiento en saque } \\
\hline Primer saque (k) & $61.5=35$ & $62.1 \pm 5.7$ & 0.6 & 0,770 \\
\hline $\begin{array}{l}\text { Puntos gansdos 1er } \\
\text { sque (b) }\end{array}$ & $759 \pm 2 . y^{*}$ & $66.9 \pm 2.7$ & 90 & 80.001 \\
\hline $\begin{array}{l}\text { Puntos grinados } 20 \\
\text { saque (b) }\end{array}$ & $54.5 \pm 3.5^{4}$ & $47.1 \pm 2.1$ & 75 & 80001 \\
\hline $\begin{array}{l}\text { Bresk points salvados } \\
\text { M) }\end{array}$ & $65.4 \pm 39^{\circ}$ & $58 \pm 2.6$ & 73 & 40.001 \\
\hline $\begin{array}{l}\text { Juegos ganados al saque } \\
\text { Wi }\end{array}$ & $95.3 \pm 37^{4}$ & $71.6 \pm 5.0$ & 137 & 40.001 \\
\hline $\begin{array}{l}\text { Puntos gandos al saque } \\
\text { (y) }\end{array}$ & $67.5 \pm 2.7^{*}$ & $513 \pm 2.2$ & b. & 40001 \\
\hline \multicolumn{5}{|l|}{ Rend imiento en resto } \\
\hline $\begin{array}{l}\text { Puntos ganados al resto } \\
\text { uer saque (h) }\end{array}$ & $30.7 \pm 2.3$ & $3 y^{2 \pm 2.2}$ & B. & 6001 \\
\hline
\end{tabular}

\begin{tabular}{|l|l|l|l|l|}
\hline Variable & Masculino & Femenino & Diff. & $p$ \\
\hline $\begin{array}{l}\text { Puntos ganados al resto } \\
20 \text { saque (\%) }\end{array}$ & $51.9 \pm 2.2$ & $57.4 \pm 2.4^{*}$ & 5.5 & 40.001 \\
\hline $\begin{array}{l}\text { Break points ganados } \\
(\%)\end{array}$ & $39.9 \pm 2.7$ & $46.9 \pm 3.4^{*}$ & 7.0 & 40.001 \\
\hline $\begin{array}{l}\text { Juegos ganados al resto } \\
(\%)\end{array}$ & $25.4 \pm 3.9$ & $40.7 \pm 4.7^{*}$ & 15.3 & $<0.001$ \\
\hline $\begin{array}{l}\text { Puntos ganados al resto } \\
(\%)\end{array}$ & $38.9 \pm 2.2$ & $46.0 \pm 2.1^{*}$ & 7.1 & 40.001 \\
\hline
\end{tabular}

Tabla 1. Comparación de medias de las parámetras de rendimiento del saque y resto del top- 8 del ranking profesional de 2017 mesculino y femenino. "Diferencios significativas a favor, pro.o1. Volores expresados en media \pm desvioción tipica.

Los resultados de la regresión lineal determinaron las variables break points salvados y juegos ganados al saque como las más influyentes sobre la posición final en el ranking masculino. Como muestra la Figura 1, un mayor porcentaje en estas variables determina una posición más elevada en el ranking masculino (puntos azules), observando diferencias del $~ 10 \%$ entre el octavo y el primer clasificado. Por lo tanto, se observa una influencia significativa en el ranking masculino (a mayor porcentaje, más alta la posición en el ranking). No se identificaron diferencias en el ranking femenino (puntos rojos).
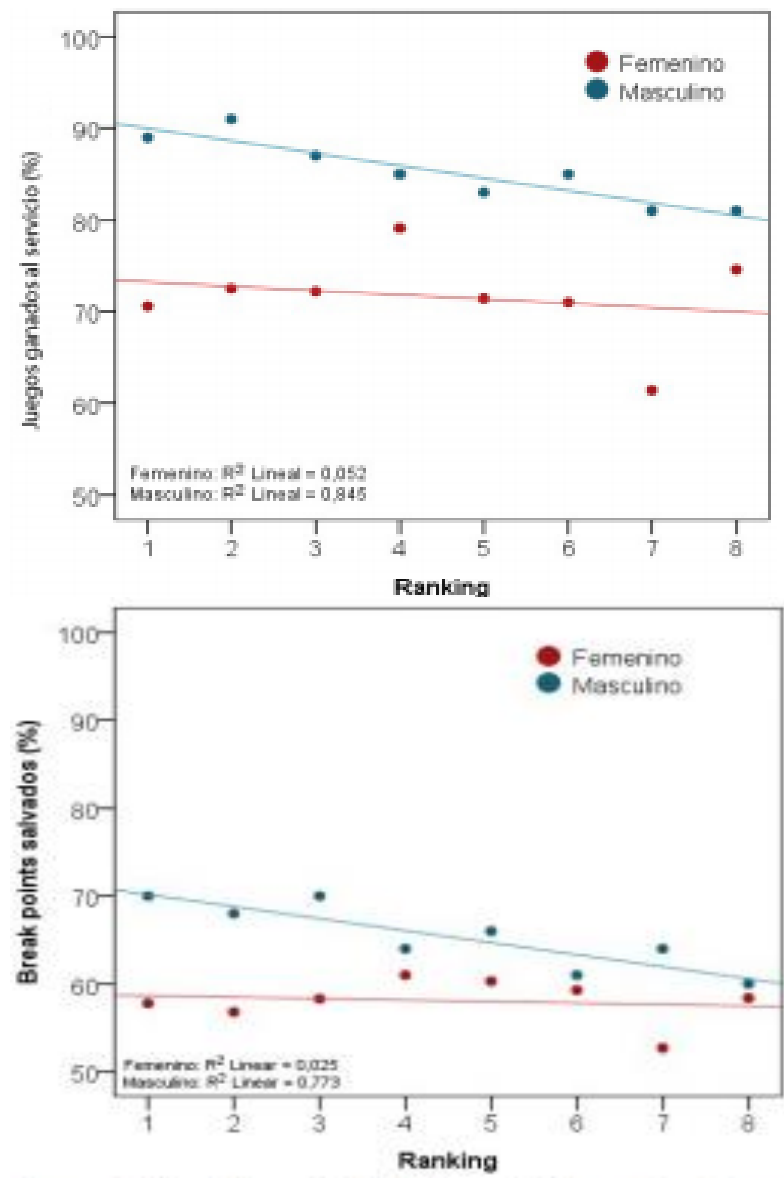

Figura 1. Grófico de dispersiän de la influencia del parcentaje de juegos ganadas al servicio (arriba) y los puntos de brealos solvados (abaja) sobre la posición en el ranking. 


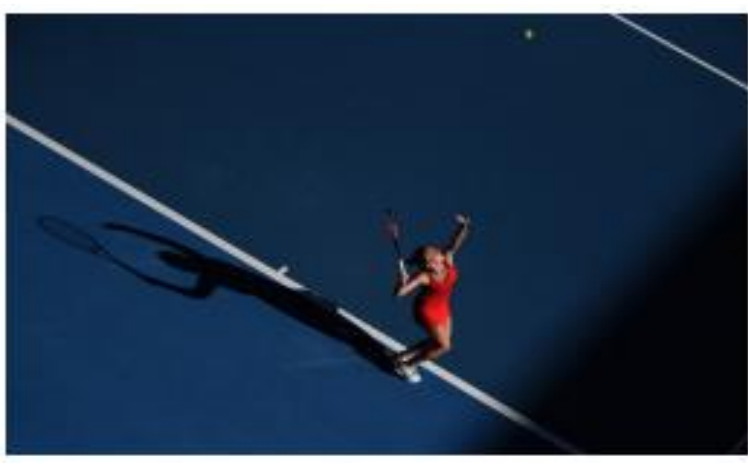

\section{DISCUSIÓN Y CONCLUSIONES}

Los resultados de este trabajo han mostrado que, tanto hombres como mujeres ganan un porcentaje mayor de puntos y juegos al saque que al resto, además de ganar más puntos al resto en el segundo saque que en el primero, siguiendo la línea del estudio de Mecheri, Rioult, Mantel, Kauffmann y Benguigui (2016). Por lo tanto, un buen porcentaje de primeros servicios parece determinante en el resultado del punto en tenis. Sin embargo, los jugadores masculinos obtienen un porcentaje significativamente mejor en los parámetros de servicio que las mujeres, mientras que estas últimas obtienen significativamente mayores porcentajes en el rendimiento del resto. Este resultado podría explicarse principalmente a una mayor velocidad del saque en hombres (Verlinden et al., 2004).

Por otro lado, los resultados de este trabajo mostraron como los jugadores con mejor ranking obtienen mejores parámetros en el servicio, resultados que siguen la línea del estudio de Barnett et al. (2008). De este modo, se han encontrado diferencias de aproximadamente un $10 \%$ en el porcentaje de break points salvados y de puntos ganados al saque entre el número 1 y el número 8 del ranking masculino de tenis. Sin embargo, las variables de saque y resto no se han mostrado como determinantes en el ranking femenino, lo que podría deberse a una mayor igualdad entre las jugadoras o una menor dependencia de estas variables en el resultado final del partido.

Por lo tanto, los resultados de este trabajo muestran los parámetros de rendimiento al servicio y resto del top-8 masculino y femenino de tenis, y que pueden servir de referencia a entrenadores y jugadores en la planificación y diseño de sus entrenamientos. Además, estos datos parecen demostrar como el servicio es un golpe muy influyente en el tenis masculino y que puede determinar la necesidad de adoptar un patrón de juego que depende de un buen saque, mientras que en el tenis femenino las jugadores pueden adaptarse a diferentes estilos de juego. Finalmente, algunos estudios han mostrado como el servicio y el resto son más determinantes en unas superficies que en otras (Brown \& O'Donoghue, 2008), por lo que futuras investigaciones podrían contemplar las posibles diferencias de estos parámetros entre superficies de juego.

\section{REFERENCIAS}

Barnett, T., Meyer, D., \& Pollard, G. (2008). La aplicación de las estadísticas del partido para aumentar el rendimiento del servicio. Medicine and Science in Tennis, 1, 2.

Brown, E., \& O'Donoghue, P. (2008). Efecto del género y la superficie en la estrategia del tenis de élite. ITF Coaching and Sport Science Review, 15(46), 11-13.

Giampolo, F., \& Levey, J. (2013). Championship tennis. Champaign, IL.: Human Kinetics.

Hughes, M., \& Franks, I.M. (2004). Notational Analysis of Sport: Systems for Better Coaching and Performance in Sport. London: Routledge. https://doi.org/10.4324/9780203641958

Mecheri, S., Rioult, F., Mantel, B., Kauffmann, F., \& Benguigui, N. (2016). The Serve Impact in Tennis: First Large-Scale Study of Big HawkEye Data. Statistical Analysis and Data Mining: The ASA Data Science Journal, 9(5), 310-325 https://doi.org/10.1002/sam.11316

O'Donoghue, P., Girad, O., \& Reid, M. (2013). Racket Sports. In T. McGarry, P. O'Donoghue \& J. Sampaio (Eds.), Routledge Handbook of Sports Performance Analysis (pp. 376-386). NY: Routledge.

O'Donoghue, P., \& Ingram, B. (2001). A notational analysis of elite tennis strategy. Journal of Sport Sciences, 19(2), 107-115. https://doi.org/10.1080/026404101300036299

Verlinden, M., Van Ruyskensvelde, J., Van Gorp, B., De Decker, S., Goossens, R. \& Clarijs, J.P. (2004). Effect of gender and tennis court surface properties upon strategy in elite singles. In: A. Lees, J.F. Kahn, I.W. Maynard. Science and Racket Sports III, (pp 163168). Routledge; Taylor \& Francis Group.

\section{CONTENIDO ITF ACADEMY RECOMENDADO (HAZ CLICK ABAJO)}

\section{TTF Academy}

Derechos de Autor (c) 2018 Bernardino Javier Sánchez-Alcaraz Martínez, Javier Courel-Ibáñez, Alejandro Sánchez-Pay y Samuel García Cambronero

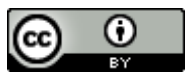

Este texto está protegido por una licencia CreativeCommons 4.0 .

Usted es libre para Compartir — copiar y redistribuir el material en cualquier medio o formato-y Adaptar el documento - remezclar, transformar y crear a partir del material- para cualquier propósito, incluso para fines comerciales, siempre que cumpla la condición de:

Atribución: Usted debe dar crédito a la obra original de manera adecuada, proporcionar un enlace a la licencia, e indicar si se han realizado cambios. Puede hacerlo en cualquier forma razonable, pero no de forma tal que sugiera que tiene el apoyo del licenciante o lo recibe por el uso que hace de la obra. 